\title{
Dialisi e bioetica
}

\section{Q. Maggiore}

Nefrologo in pensione, Firenze

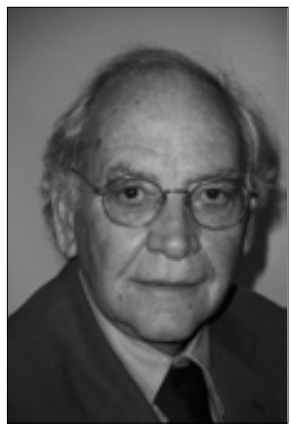

Ho molto apprezzato l'iniziativa del gruppo nefrologico torinese di pubblicare su questo giornale una ricerca dedicata ai problemi di bioetica connessi con la terapia sostitutiva renale. In questo articolo cercherò di inquadrare l'argomento affrontato dai torinesi, raccontandone brevemente la storia e le implicazioni che sono oggetto di vivace dibattito non solo nei periodici scientifici più importanti ma anche, e soprattutto, nei giornali di informazione.

I problemi etici che riguardano la medicina vengono oggi indicati con il termine bioetica. La bioetica esiste solo da pochi anni, ma è talmente cresciuta di importanza da venire inclusa come materia di insegnamento universitario nelle facoltà di Medicina, Biologia e Filosofia in diversi Paesi del mondo. Albert Jonsen, professore di Bioetica presso l'Harvard University di Boston, ha scritto che la bioetica è nata il 9 marzo 1960, quando il Dottor Belding Scribner cominciò a dializzare a Seattle il signor Clyde Shields, un paziente (professione macchinista) affetto da uremia terminale. L'esperienza di Seattle inaugurò l'era dei trattamenti tecnologici atti a sostituire la funzione di organi vitali irrimediabilmente compromessi dalla malattia. All'inizio la disponibilità di risorse per il trattamento sostitutivo renale era così scarsa da consentirne la fruibilità solo a pochissimi dei pazienti che in fila interminabile si presentavano a Seattle nella speranza di essere salvati dalla morte. Come scegliere i pochissimi fra i tanti che lo chiedevano? Allo scopo di evitare arbitrii e favoritismi fu istituita una Commissione non medica composta da varie figure professionali, ma senza nefrologi o internisti (ne faceva parte solo un chirurgo), che elaborarono una serie di criteri per scegliere i malati da trattare fra quelli che ne avevano urgente bisogno. I criteri erano basati fondamentalmente sull'età (minore di 45-50 anni), sul ruolo sociale del paziente (ad esem- pio l'imprenditore veniva preferito al dipendente), sulla capacità di pagarsi il trattamento sostitutivo, sull'assenza di comorbidità gravi ecc. Questo metodo di selezione, che in quelle circostanze probabilmente non aveva alternative, stravolgeva i canoni etici della medicina tradizionale, basati sul paternalismo medico che a sua volta si richiamava alla tradizione ippocratica e all'insegnamento espresso nella parabola evangelica del Buon Samaritano.

La novità rivoluzionaria di questo approccio destò una vivissima reazione nell'opinione pubblica, espressa in un memorabile articolo, pubblicato nel novembre 1962 su Time Magazine a firma di Shana Alexander, intitolato "They decide who dies, who live". Alla Commissione di Seattle fu affibbiato il nomignolo tra lo spregiativo e il derisorio di "God Commission" (la Commissione del Padreterno), tanto che sotto la pressione dell'opinione pubblica essa venne sciolta e si adottarono nuovi criteri basati sulla regola del "first come-first serve", e sulla capacità di pagarsi il trattamento direttamente o tramite compagnie assicurative private. Continuavano ad essere esclusi, decretandone in pratica la condanna a morte, $\mathrm{i}$ pazienti anziani, quelli con importanti malattie associate e, comunque, tutti coloro che non erano in grado di sostenere le spese del trattamento. Il protrarsi di tale situazione provocò una reazione così forte nell'opinione pubblica che alla fine, nel 1973, 13 anni dopo l'evento di Seattle, il Congresso americano emanò una legge $(\mathrm{Pu}-$ blic Law 92-603) che consentiva, solo ed esclusivamente agli uremici cronici, il diritto di usufruire gratuitamente delle cure dialitiche sotto la copertura assicurativa di Medicare e Medicaid. Questa legge non aveva precedenti nella storia americana e bisognerà aspettare l'avvento della presidenza Obama perché provvidenze analoghe fossero adottate nel 2010 a favore di pochi altri settori dell'assistenza medica.

Con l'abbattimento delle barriere economiche, vennero a cadere praticamente tutti i criteri medici di esclusione; 
in particolare non precludevano l'accesso alla dialisi né l'età avanzata, né la coesistenza di condizioni morbose anche gravi. Come conseguenza della liberalizzazione all'accesso ci sono attualmente centinaia di migliaia di pazienti con uremia terminale che vengono trattati con la dialisi nei Paesi delle società cosiddette affluenti. Oggi il trattamento dialitico viene fruito dagli anziani in misura relativamente molto più alta che dai giovani: nella popolazione generale le persone con età superiore ai 65 anni sono il 14\%, mentre nella popolazione dialitica essi rappresentano circa il $45 \%$, cioè oltre tre volte di più. Gli anziani bisognosi del trattamento dialitico aumentano di anno in anno con un ritmo maggiore rispetto alle altre fasce di età: i pazienti ultrasessantacinquenni entrati in dialisi in Italia nel 1985 erano 3979, ma già nel 1991 erano diventati 5154 (un aumento di quasi il 30\%). Le previsioni demografiche indicano che nei prossimi 50 anni gli anziani aumenteranno ancor più nella popolazione generale e ciò si ripercuoterà inevitabilmente sulla composizione anagrafica della popolazione dialitica con un incremento proporzionalmente maggiore dei pazienti ultrasettantenni.

Proviamo ora a immaginare che cosa avverrebbe oggi se applicassimo i criteri di selezione basati sull'età. Con un limite fissato a 50 anni, come avveniva negli anni Sessanta, escluderemmo il $92,7 \%$ della popolazione che attualmente entra in dialisi. Alzando il limite a 65 anni, come avveniva in gran parte dei Centri dialisi negli anni Settanta, ne escluderemmo il 73,7\%.

D'altra parte, non meno impressionanti sono state le conseguenze della "deselezione" verificatasi dopo l'emanazione della Legge 92-603: esse sono eloquentemente espresse dalla moria per abbandono o per rifiuto della terapia dialitica che ha interessato dal $20 \%$ al $25 \%$ della popolazione dialitica, soprattutto nelle sue fasce di età più avanzata. Questi sono malati che per le sofferenze intollerabili causate da malattie coesistenti o dalle complicanze del trattamento stesso sospendono la dialisi di loro volontà, se sono ancora capaci di intendere o volere, o se queste capacità appaiono totalmente compromesse, su sollecitazione dei familiari o dei medici curanti. Stando ai censimenti, il fenomeno della sospensione del trattamento sembra essere meno frequente in Europa (comunque una segnalazione in Francia la stima intorno al 20\%) che negli Stati Uniti e in Canada; ma non si può escludere che si tratti solo di apparenza, che, cioè, i medici europei siano più riluttanti a dichiararla esplicitamente nei questionari. La questione non è poi così importante, quello che conta è che una proporzione non trascurabile degli anziani non trae beneficio da questo trattamento e il motivo è di una ovvietà lampante: con la dialisi noi sostituiamo la funzione di un organo vitale come il rene, ma se vi sono altri organi vitali gravemente ammalati, come spesso succede negli anziani, il trattamento sostitutivo renale non potrà né arrestare né rallentare l'evoluzione di quelle malattie verso l'esito infausto, né tanto meno migliorarne la qualità di vita. Al contrario, il trattamento sostitutivo finirà per procurare maleficio invece che beneficio al paziente, oltre a comportare un ingiusto spreco di risorse che potrebbero essere, invece, più proficuamente indirizzate a soddisfare altri bisogni della collettività. Quello che da anni avviene nella popolazione dialitica sta inverando la profezia di Ivan Illich:

«La medicina non può far molto per $i$ mali che si accompagnano alla senescenza... Di massima, le cure dei vecchi che comportano un intervento professionale non soltanto aggravano le loro sofferenze ma, se l'intervento riesce, le protraggono...

Quando il compito di assistere o di guarire passa alle organizzazioni o alle macchine, è inevitabile che la terapia diventi un rituale incentrato sulla morte.

Ma la nemesi trascende anche il sacrificio umano. La conseguenza estrema della nemesi medica è l'espropriazione della morte».

La domanda che ci si deve porre, allora, è la seguente: non sarebbe stato meglio abbandonare questi pazienti alla loro sorte invece di prolungare la loro agonia con la dialisi? Non sarebbe meglio selezionare i pazienti in modo da riservare questa risorsa terapeutica, enormemente costosa, solo a coloro che ne possano trarre beneficio, escludendo quelli in cui questo risultato è prevedibilmente del tutto improbabile? In altre parole, non è meglio selezionare prima ("treatment withholding") che sospendere dopo ("treatment withdrawing") un trattamento che ha come unico risultato quello di prolungare l'agonia del paziente, arrecandogli male invece che bene? L'approccio selettivo sarebbe perfettamente in linea con canoni della bioetica quali: beneficenza, non maleficenza, autonomia, giustizia. Il compito, però, è tutt'altro che facile. Occorre ricordare che i criteri che ci guidano per formulare la prognosi sono basati essenzialmente su statistiche osservazionali che, pertanto, possono risultare del tutto fallaci quando vengono applicati al singolo paziente. Per questa ragione, salvo forse i casi di demenza o di metastasi cancerose diffuse, ogni paziente candidato è teoricamente eleggibile per il trattamento sostitutivo. In presenza di fattori di rischio che possano pregiudicarne il risultato, il medico ha il dovere di prospettare al paziente tutte le possibili implicazioni inerenti alla terapia sostitutiva, anche alla luce del contesto familiare, della rete di supporto sociosanitario ecc. La decisione finale spetta sempre e comunque al pazien- 
te. Il rispetto dell'autonomia del paziente, se "competente", è un criterio accettato pressoché universalmente, ed è sancito dalla nostra Costituzione.

In sintesi i problemi bioetici collegati con il trammento dialitico sono i seguenti:

- È etica la selezione dei pazienti su base clinica?

- Il paziente è capace di dare il consenso informato?

- C'è qualcuno che può surrogarlo?

- Ha espresso direttive per le cure di fine-vita?

- Quando la dialisi serve solo a prolungare il processo del morire, è lecito sospenderla?

- È lecito cessare la dialisi in un paziente incompetente anche contro il parere dei familiari?

- Ci sono opzioni alternative alla dialisi?

- Chi decide?

Fin dal 1999 la Società americana di Nefrologia ha formalizzato in linee guida l'invito ai pazienti di formulare le cosiddette "living will" o "advance directives" nel momento di entrare in un programma di terapia sostitutiva (Tab. I). Le direttive possono essere specificate per scritto oppure il paziente può nominare un suo rappresentante delegato a prendere le decisioni nel caso di sua sopravvenuta incompetenza. Nei casi più gravi viene offerta, in alternativa al trattamento sostitutivo, l'opzione delle cure palliative che includono il trattamento conservativo che si è dimostrato non in- feriore ad esso in termini di beneficio sintomatico e di sopravvivenza. Per aiutare la scelta consapevole, le linee guida prevedono un periodo di prova del trattamento dialitico.

"Clinical Practice Guideline on Shared Decision-Making in the Appropriate Initiation of and Withdrawal from Dialysis" Renal Physicians Association and American Society of Nephrology. Washington, DC November 1999.

- Decisioni condivise

- Consenso (o Rifiuto) informato

- Stima della prognosi

- Risoluzione dei conflitti

- Directive anticipate

- "Withholding" o "Withdrawing" la Dialisi

- Pazienti speciali

- Prova per un periodo di tempo limitato

- Cure Palliative.

Negli anni Settanta i nefrologi italiani (medici e infermieri) hanno svolto storicamente un ruolo decisivo per affermare il diritto degli uremici a ricevere le cure salvavita. Noblesse oblige: tocca ai nefrologi italiani di oggi essere in prima linea nel difendere il diritto dei malati a una morte dignitosa. Le linee guida americane sono secondo me un buon modello da cui partire.

Indirizzo degli Autori:

Professor Quirino Maggiore

Via delle Fonti 62

50015 Firenze

maggiore_quirino@libero.it 